\title{
REMARKS ON FUNCTIONS OF BOUNDED MEAN OSCILLATION ON RIEMANN SURFACES
}

To Professor Mitsuru Ozawa on the occasion of his 60th birthday

By Yukio Kusunoki and Masahiko Taniguchi

\section{Introduction.}

The functions of bounded mean oscillation in the euclidean spaces have been extensively studied from various points of view (cf. for instance [1], [4]). The present article is also concerned with those functions on Riemann surfaces, and actually we show that some fundamental potential functions on Riemann surfaces are of bounded mean oscillation, and moreover we discuss some related topics.

First we show in $\S 1$ that a harmonic function $u$ with finite Dirichlet integral on a given Riemann surface is of bounded mean oscillation if it can be written as $c \cdot \log |f|$ with some analytic function $f$, (in other words, if every periods of the conjugate harmonic function of $u$ is equal to zero modulo a constant,) which generalizes Metzger's result [3].

The next $\S 2$ and $\S 3$ are concerned with functions of bounded mean oscillation in two dimensions. We shall prove that every Green's potential with the measure having a compact support is of bounded mean oscillation, and so are every potentials with the measure having a finite total mass in the unit disk and every harmonic function with finite Dirichlet integral on Riemann surfaces of finite type. Finally we shall give a sufficient condition under which a function of bounded mean oscillation is of bounded mean oscillation in hyperbolic metric.

\section{$\S 1$. On harmonic functions of BMO.}

Let $U$ be the unit disk $\{|z|<1\}$ and $T=\{|z|=1\}$. An integrable function $g(t)$ on $T$ is, by definition, of bounded mean oscillation if

$$
\sup _{I} \frac{1}{|I|} \int_{I}\left|g(t)-g_{I}\right| d t<+\infty
$$

where $|I|=\int_{I} d t, g_{I}=\frac{1}{|I|} \int_{I} g(t) d t$ and the supremum is taken for all subarcs $I$ in $T$. Set

Received March 7, 1983 
$\mathrm{BMOH}(U)=\{u: u$ is the Poisson integral of some function of bounded mean oscillation on $T\}$.

Next for every Riemann surface $R$ having $U$ as a universal covering surface, we set

$$
\begin{aligned}
\operatorname{BMOH}(R)= & \{u: u \text { is a harmonic function on } R \text { such that } \\
& u \circ \pi \text { belongs to } \operatorname{BMOH}(U)\},
\end{aligned}
$$

where $\pi$ is a universal covering mapping from $U$ onto $R$.

Remark. The space $\mathrm{BMOH}(R)$ does not depend on the choice of $\pi$. Actually, $\operatorname{BMOH}(R)$ is coincident with the space of harmonic functions $u(p)$ on $R$ for which $\sup _{q \in R} \iint_{R}|\operatorname{grad} u(p)|^{2} g(p, q)|d p \wedge d \bar{p}|$ is finite, when $R$ admits Green's functions $g(p, q)$ (cf. [3] $\S 2$ Remark).

We denote by $\operatorname{HD}(R)$ the space of all harmonic functions on $R$ with finite Dirichlet integral, and by $\mathrm{BMOA}(R)$ and $\mathrm{AD}(R)$, respectively, the subspaces of $\mathrm{BMOH}(R)$ and $\mathrm{HD}(R)$ consisting of analytic functions on $R$. Without loss of generality, we shall consider in the sequel only real-valued harmonic functions.

It is known ([3] Theorem 1) that $\mathrm{AD}(R)$ is contained in $\operatorname{BMOA}(R)$. Here our problem is to find a relationship between $\operatorname{HD}(R)$ and $\mathrm{BMOH}(R)$. A partial answer is the following

THEOREM 1. Let $R$ be a Riemann surface having $U$ as a unversal covering surface and $u \in \operatorname{HD}(R)$. Suppose that $u=c \cdot \log |f|$ with some real constant $c$ and an analytic function $f$ on $R$, then $u$ belongs to $\mathrm{BMOH}(R)$.

In other words, $u$ in $\mathrm{HD}(R)$ belongs to $\mathrm{BMOH}(R)$ if all the periods of the conjugate differential $* d u$ of $d u$ along loops on $R$ are equal to zero modulo a constant.

Proof. Let $\pi$ be a universal covering mapping from $U$ onto $R$, then $V(z)$ $=u \circ \pi(z)$ is a harmonic function on $U$. Consider a holomorphic function $F(z)$ on $U$ whose real part is $V(z)$ (for instance, $F(z)=c \cdot(\log f) \circ \pi(z)$ ). Let $G$ be the Fuchsian group associated with $R$ and $\pi$, then from the assumption it holds that

$$
F(\gamma(z)) \equiv F(z) \quad(\bmod .2 \pi i c)
$$

for every $\gamma$ in $G$, where $i=\sqrt{-1}$.

We shall study about the image domain $F(U)$ of $U$ by $F$ in the complex $w$ plane. Let $\omega$ be the fundamental domain of $G$. on $U$ (given in [5] XI $\S 2$ ), then $F(U)$ is contained in the union of $\{F(\omega)+2 \pi i s c\}_{s=-\infty}^{+\infty}$. For every real number $t$ we denote by $L_{t}$ and $L(t)$ the set $F(\omega) \cap\{w ; \operatorname{Re} w=t\}$ and its length $\left|L_{t}\right|$ respectively. Since the Dirichlet integral $D(u)$ of $u$ on $R$ is finite, it holds that 


$$
\begin{aligned}
\int L(t) d t & =\text { area }|F(\omega)| \text { of } F(\omega) \leqq \iint_{\omega}\left|F^{\prime}(z)\right|^{2} d x d y \\
& =D(u)<+\infty .
\end{aligned}
$$

For every positive number $\delta$, let $e(\delta)=\{t: L(t) \leqq \delta\}$ and $e^{\prime}(\delta)=\{t: L(t)>\delta\}$, then $\left|e^{\prime}(\delta)\right| \leqq D(u) / \delta<+\infty$. Consider a sequence $\left\{I_{n}\right\}_{n=-\infty}^{+\infty}$ of disjoint intervals on the real axis such that the length $\left|I_{n}\right|=1$ and the distance between $I_{n-1}$ and $I_{n}$ is equal to 1 for every $n$. From the above fact one sees that there is a positive integer $n_{0}=n_{0}(\delta)$ such that for all $n$ with $|n| \geqq n_{0}$

$$
\left|e^{\prime}(\delta) \cap I_{n}\right| \leqq 1 / 2 \text {, hence }\left|e(\delta) \cap I_{n}\right| \geqq 1 / 2 \text {. }
$$

Now fix $\delta$ and $n$ with $|n| \geqq n_{0}$. Consider the set

$$
e_{n}=\left\{L_{t}: t \in e(\grave{\partial}) \cap I_{n}\right\}
$$

and all shifts $e_{n}+2 \pi i s c$, where $s=0, \pm 1, \pm 2, \cdots$. Then we claim that for every square $Q$ of side length $q$ placed parallel to the coordinate axes, we have

$$
\sum_{s=-\infty}^{+\infty}\left|\left(e_{n}+2 \pi \imath s c\right) \cap Q\right| \leqq \nu\left|e_{n} \cap \tilde{Q}\right|,
$$

where $\nu=[q / 2 \pi|c|]+1$ and $\tilde{Q}$ is the strip region of width $q$ which is parallel to the imaginary axis and contains $Q$.

To see this, let $\chi_{Q}$ be the characteristic function of $Q$. Then $\left|e_{n} \cap Q\right|=$ $\int_{e_{n}} \chi_{Q}(w) d m(w), d m(w)$ being the Lebesgue measure, and it holds that

$$
\begin{aligned}
\left|\left(e_{n}+2 \pi i s c\right) \cap Q\right| & =\int_{e_{n}+2 \pi \imath s c} \chi_{Q}(w) d m(w) \\
& =\int_{e_{n}} \chi_{Q-2 \pi \imath s c}(w) d m(w) .
\end{aligned}
$$

Since the shifts $Q-2 \pi \imath s c$ of $Q$ mutually overlap at most $\nu$ times, it follows that

$$
\sum_{s=-\infty}^{+\infty} \chi_{Q-2 \pi \imath s c}(w) \leqq \nu
$$

for every $w$ in $\tilde{Q}$, hence by the equality above we have the inequality $\left(^{*}\right)$.

From $(*)$ it follows that for the set

$$
E_{n}=\left\{w \in F(U): \operatorname{Re} w \in e(\delta) \cap I_{n}\right\}
$$

we have

$$
\left|E_{n} \cap Q\right| \leqq \nu\left|e_{n} \cap \tilde{Q}\right| .
$$

While $\left|e_{n} \cap \tilde{Q}\right|<\delta$ and $\left|e(\delta) \cap I_{n}\right| \geqq 1 / 2$ for every $n$ with $|n| \geqq n_{0}$, so if we take $q \geqq 4 n_{0}+4$, there is an $n$ with $|n| \geqq n_{0}$ such that $\tilde{Q} \supset I_{n}$. Therefore we have 


$$
|Q \backslash F(U)| \geqq \frac{q}{2}-\left|E_{n} \cap Q\right| \geqq \frac{q}{2}-\delta \nu,
$$

so $|Q \backslash F(U)| \geqq q / 4 \geqq 1$ provided that $\delta<1 / 4((1 / 2 \pi|c|)+1)(<q / 4 \nu)$.

Every disk $\Delta\left(w_{0}\right)=\left\{w:\left|w-w_{0}\right| \leqq q\right\}$ with center $w_{0} \in F(U)$ contains such a square $Q$ as above. Thus for every $w_{0} \in F(U)$, we have

$$
\begin{aligned}
\operatorname{cap}\left(\Delta\left(w_{0}\right) \backslash F(U)\right) & \geqq\left(\left|\Delta\left(w_{0}\right) \backslash F(U)\right| / \pi e\right)^{1 / 2} \\
& \geqq 1 /(\pi e)^{1 / 2}>0 .
\end{aligned}
$$

(For the first inequality, see [5] Theorem III. 10). Hence by a theorem due to Hayman and Pommerenke ([2] Theorem 1), we know that $F(z)$ belongs to $\operatorname{BMOA}(U)$, which implies that $u \in \operatorname{BMOH}(R)$.

q.e.d.

Actually we have shown the following

PROPOSITION 1. Let $\int$ be an analytıc function on $R$ such that $\iint_{R}\left|f^{\prime} / f\right|^{2} d x d y$ $<+\infty$, then $(\log f) \circ \pi$ belongs to $\operatorname{BMOA}(U)$.

Applying Proposition 1 to $f=\exp g$ with $g \in \mathrm{AD}(R)$, we know that $\operatorname{AD}(R)$ $\subset \operatorname{BMOA}(R)$ (Metzger [3]).

Another result concerning harmonic functions with finite Dirichlet integral will be given in the next section.

\section{$\S 2$. On potentials of BMO.}

Analogously as in $\S 1$, a locally integrable function $f(z)$ on $U$ belongs, by definition, to $\operatorname{BMO}(U)$ if

$$
\sup _{B} \frac{1}{|B|} \int_{B}\left|f(z)-f_{B}\right| d m(z)<+\infty
$$

where $|B|=\int_{B} d m, f_{B}=\frac{1}{|B|} \int_{B} f d m$ and the supremum is taken for all closed disk $B$ in $U$. For a Riemann surface $R$ having $U$ as a universal covering surface, set

$$
\begin{aligned}
\operatorname{BMO}(R)=\{ & \{f: f \text { is a function on } R \text { such that } \\
& f \circ \pi \text { belongs to } \operatorname{BMO}(U)\}
\end{aligned}
$$

Here $\pi$ is a universal covering mapping from $U$ onto $R$, and $\operatorname{BMO}(R)$ does not depend on the choice of $\pi$ (cf. [4] 9p). In this section we shall show first the following

THEOREM 2. If $R$ admits Green's function, then every Green's potential 
$P^{\mu}(p)=\int_{R} g(p, q) d \mu(q)$ with measure $\mu$ having a compact support on $R$ belongs to $\operatorname{BMO}(R)$, where $g(p, q)$ is the Green's function on $R$ with pole $q$.

To prove Theorem 2, we consider an auxiliary function. Fix $q \in R$ arbitrarily, and let $\pi_{q}$ be a universal covering mapping such that $\pi_{q}(0)=q$. Also fix a positive number $t_{q}$ such that $\left\{|z|<3 t_{q}\right\}$ is mapped univalently by $\pi_{q}$, and set

$$
H_{t, q}(z)=\sum_{\gamma \in G_{q}} \log ^{+}\left(t_{q} /|\gamma(z)|\right),
$$

where $G_{q}$ is the Fuchsian group associated with $R$ and $\pi_{q}$. Then $H_{t, q}$ is $G_{q^{-}}$ invariant, and it is known ([4] 10p) that $H_{t, q^{\circ}} \pi_{q}^{-1}$ considered as a function on $R$ belongs to $\operatorname{BMO}(R)$.

LEMma. Let $R$ and $g(p, q)$ be as in Theorem 2 , then $g(p, q)$ belongs to $\operatorname{BMO}(R)$.

Proof. It is well-known ([5] Theorem XI. 13) that

$$
g\left(\pi_{q}(z), q\right)=\sum_{\gamma \in G_{q}} \log (1 /|\gamma(z)|) .
$$

Now fix $t_{q}$ as above, then $g(p, q)-H_{t, q^{\circ}} \pi_{q}^{-1}(p)$ is bounded on $R$, hence belongs to $\mathrm{BMO}(R)$. Thus from the above remark we have the assertion.

Proof of Theorem 2. Let $E$ be the compact support of $\mu$ on $R$, then we can take a positive constant $t$ as $t_{q}$ for every $q \in E$. Let $H_{t, q}(z)$ and $\pi_{q}$ be as above and set $h_{q}(p)=H_{t, q^{\circ}} \pi_{q}^{-1}(p)$ for every $q \in E$. Next fix any point $q$ in $E$ and denote a fixed $\pi_{q}$ simply by $\pi$. Consider the set $F=\pi^{-1}(E) \cap \omega$, where $\omega$ is as in the proof of Theorem 1 . Then clearly $F$ is relatively compact and $\pi$ is bijective from $F$ onto $E$. For every $\alpha \in F$, set $H_{\alpha}(z)=h_{q^{\prime}} \circ \pi(z)$, where $q^{\prime}=\pi(\alpha)$. Then we can see that there is a $K$ satisfying the following condition; for every $q^{\prime} \in E$, there is a $K$-quasiconformal selfmapping of $R$ which is homotopic to the identical mapping and maps $\pi(\{0<|z|<t\})$ conformally onto $\pi_{q^{\prime}}(\{0<|z|<t\})$. Equivalently, for every $\alpha \in F$, there is a $K$-quasiconformal selfmapping $F_{\alpha}(z)$ of $U$ such that $F_{\alpha}(0)=\alpha, \gamma \circ F_{\alpha}=F_{\alpha} \circ \gamma$ for every $\gamma \in G\left(=G_{q}\right)$ and $H_{\alpha} \circ F_{\alpha}(z) \equiv H_{0}(z)$. Since $h_{q^{\prime}}$ belongs to $\mathrm{BMO}(R)$ for every $q^{\prime} \in E$ as noted above, using Reimann's theorem ([4] V.C. Satz 3) we can find an $M$ depending only on $K$ and $h_{q}$ such that

$$
\int_{B}\left|H_{\alpha}(z)-a_{B}(\alpha)\right| d m(z) \leqq M \cdot|B|
$$

for every $\alpha$ in $F$ and every closed disk $B$ in $U$, where $a_{B}(\alpha)=\frac{1}{|B|} \int_{B} H_{\alpha}(z) d m(z)$. Also it is easily seen that for a fixed $B, a_{B}(\alpha)$ is continuous with respect to $\alpha$, 
hence in particular, $a_{B}=\int_{F} \alpha_{B}(\alpha) d \mu \circ \pi(\alpha)$ is finite. Hence, letting $H^{\mu} \circ \pi(z)=$ $\int_{F} H_{\alpha}(z) d \mu \circ \pi(\alpha)$, we have

$$
\begin{aligned}
& \int_{B}\left|H^{\mu} \circ \pi(z)-a_{B}\right| d m(z) \\
\leqq & \int_{B}\left(\int_{F}\left|H_{\alpha}(z)-a_{B}(\alpha)\right| d \mu \circ \pi(\alpha)\right) d m(z) \\
\leqq & M \cdot|B| \cdot \mu(E),
\end{aligned}
$$

namely, $H^{\mu}(p)$ belongs to $\mathrm{BMO}(R)$.

Finally, because $g(p, \pi(\alpha))-H_{\alpha}^{\circ} \pi^{-1}(p)$ is uniformly bounded on $R$ for every $\alpha$ in $F, P^{\mu}(p)-H^{\mu}(p)$ is bounded on $R$, hence belongs to $\operatorname{BMO}(R)$. Thus we conclude that $P^{\mu}(p)$ belongs to $\mathrm{BMO}(R)$.

q.e.d.

Remark. In the case that $R=U$, we can show that every potential with measure of totally finite mass belongs to $\operatorname{BMO}(R)$. (See Proposition 2 in the next section.)

Relating of Theorem 1 in $\S 1$, we note the following

THEOREM 3. Let $R$ be a Riemann surface of finite type and $u(p) \in \operatorname{HD}(R)$. Then $u(p)$ belongs to $\mathrm{BMO}(R)$.

Proof. Let a universal covering mapping $\pi$ from $U$ onto $R$ be fixed and $R^{*}$ be the interior of the compact bordered Riemann surface obtained from $R$ by filling all punctures. Take a subsurface $S$ of $R$ which is relatively compact in $R^{*}$ and contains a neighbourhood of each puncture of $R$, and set $D=\pi^{-1}(S)$. Since $u(p)$ can be extended harmonically onto all punctures of $R, \sup _{p \in S}|u(p)|$ is finite. Also it is easily seen that there is a $K$ such that for every closed disk $B$ in $U$, not contained in $D$, with hyperbolic radius (i.e. radius with respect to the Poincaré hyperbolic metric) not greater than $K, \pi$ is injective on $B$. Here recall that there is an $r(K)(<1 / 2)$ such that every disk $B$ in $U$ with center $z_{0}$ and radius $\left(1-\left|z_{0}\right|\right) \cdot 2 r(K)$ has a hyperbolic radius not greater than $K$. Fix such an $r(K)$ once for all.

Now to prove the assertion, it suffices to find an $M$ (cf. [4] I. D. 2) such that for every closed disk $B$ with center $z_{0}$ and radius not greater than $\left(1-\left|z_{0}\right|\right) \cdot r(K)$, it holds that

$$
\int_{B}\left|V(z)-V_{B}\right| d m(z) \leqq M \cdot|B|
$$

where $V(z)=u \circ \pi(z)$. If $B$ is contained in $D$, then it is clear that 


$$
\int_{B}\left|V(z)-V_{B}\right| d m(z) \leqq 2 \cdot|B| \cdot \sup _{p \in S}|u(p)| .
$$

If $B$ is not contained in $D$, then because $\left\{w:\left|w-z_{0}\right|<2 r_{0}\right\}$ with $r_{0}=\left(1-\left|z_{0}\right|\right)$. $r(K)$ is mapped univalently by $\pi$, for every $z \in B$ it holds that

$$
\begin{aligned}
&\left|V(z)-V\left(z_{0}\right)\right|^{2} \leqq\left(\int_{0}^{r_{1}}\left|\frac{\partial V}{\partial r}\left(r e^{i \theta}+z_{0}\right)\right| d r\right)^{2} \\
& \leqq r_{1} \cdot \int_{0}^{r_{1}}\left|\frac{\partial V}{\partial r}\left(r e^{i \theta}+z_{0}\right)\right|^{2} d r \\
& \leqq r_{1} \cdot \int_{0}^{r_{1}}\left(\frac{1}{\pi r_{0}^{2}} \cdot \iint_{\left\{\mid w-z_{0}-r e^{\left.i \theta_{\mid} \leqq r_{0}\right\}}\right.}\left|\frac{\partial V}{\partial r}(w)\right|^{2} d m(w)\right) d r \\
& \leqq r_{1}^{2} \\
& \pi r_{0}^{2}
\end{aligned}
$$

where $r_{1}=\left|z-z_{0}\right|$ and $D(u)$ is the Dirichlet integral of $u$ on $R$. Since $V_{B}=V\left(z_{0}\right)$, we conclude that

$$
\int_{B}\left|V(z)-V_{B}\right| d m(z) \leqq(D(u) / \pi)^{1 / 2} \cdot|B| .
$$

Thus we have the assertion.

q.e.d.

\section{$\S 3$. On functions of BMO in hyperbolic metric.}

We consider the space $\operatorname{BMO}(R, \lambda)$ of functions of bounded mean oscillation with respect to the Poincaré hyperbolic metric $d \sigma(z)=\lambda(z) d m(z)$ with $\lambda(z)=$ $\left(1-|z|^{2}\right)^{-2}$, which is defined by using $d \sigma$ instead of $d m$ in the definition of $\mathrm{BMO}(R)$. It is known ([4] 10p) that $\operatorname{BMO}(R, \lambda)$ is contained in $\operatorname{BMO}(R)$. On the other hand, it seems to be unknown whether $\operatorname{BMO}(R, \lambda)$ is coincident with $\mathrm{BMO}(R)$. Here we show the following partial answer.

THEOREM 4. If $f$ belongs to $\mathrm{BMO}(R)$ and is bounded outside a compact set of $R$, then $f$ belongs to $\operatorname{BMO}(R, \lambda)$.

Proof. Fix a positive number $d$ arbitrarily. If the hyperbolic radius of a closed disk $B$ is less than $d$, then we can easily see that $|B| \bar{\lambda}^{-1} \cdot \int_{B}\left|F(z)-a_{B}\right| d \sigma(z)$ is bounded by a constant depending only on $f$ and $d$, where $F(z)=f \circ \pi(z),|B|_{\lambda}$ $=\int_{B} d \sigma$ and $a_{B}=|B|^{-1} \cdot \int_{B} F(z) d m(z)$, because $f \in \mathrm{BMO}(R)$ and $\sup _{z \in B} \lambda(z) / \inf _{z \in B} \lambda(z)$ is clearly bounded by a constant depending only on $d$.

Next let a positive $\varepsilon$ be arbitrarily fixed, and set 


$$
M_{s}(z)=\frac{1}{|B[z]|_{\lambda}} \cdot \int_{B[z]}|F(w)| d \sigma(w)
$$

where $B[z]$ is the disk $\{w:$ the hyperbolic distance $d(w, z) \leqq \varepsilon\}$. Then from the local integrability of $F, M_{\varepsilon}(z)$ is finite for every $z$ and continuous by the Lebesgue's convergence theorem. Clearly, $M_{\varepsilon}(z)$ is $G$-invariant (for $G$ see $\S 1$ ), and $\sup _{z \in U} M_{\varepsilon}(z)$ is finite, for $f$ is bounded outside a compact set of $R$. Also for every closed disk $B$ in $U$ the following inequality holds;

$$
\frac{1}{|B|_{\lambda}} \cdot \int_{B}|F(z)| d \sigma(z) \leqq \frac{1}{|B|_{\lambda}} \cdot \int_{B_{\varepsilon}} M_{\varepsilon}(z) d \sigma(z)
$$

where $B_{s}=\{z \in U: d(a, z) \leqq r+\varepsilon\}$ if $B=\{z \in U: d(a, z) \leqq r\}$.

In fact, we have

$$
\begin{aligned}
\int_{B_{\varepsilon}} M_{\varepsilon}(z) d \sigma(z) & =A \cdot \int_{U}\left(\int_{U}|F(w)| \cdot \chi_{B[z]}(w) d \sigma(w)\right) \chi_{B_{\varepsilon}}(z) d \sigma(z) \\
& =A \cdot \iint_{U \times U}|F(w)| \cdot \chi_{\{(z, w): d(a, z) \leqq r+\varepsilon, d(z, w) \leq \varepsilon]} d \sigma(z) d \sigma(w) \\
& \geqq A \cdot \iint_{U \times U}|F(w)| \cdot \chi_{\{(z, w): d(a, w) \leq r, d(z, w) \leqq \varepsilon]} d \sigma(z) d \sigma(w) \\
& =A \cdot \int_{U}\left(\int_{U} \chi_{B[w]}(z) d \sigma(z)\right) \cdot|F(w)| \cdot \chi_{B}(w) d \sigma(w) \\
& =\int_{B}|F(w)| d \sigma(w),
\end{aligned}
$$

where $A=1 /|B[z]|_{\lambda}$ is a constant depending only on $\varepsilon$.

Now we take $\varepsilon=d$, then $\left|B_{d}\right|_{\lambda} /|B|_{\lambda}$ is bounded by a constant $C(d)$ depending only on $d$ for every closed $\operatorname{disk} B$ in $U$ whose hyperbolic radius is not less than $d$. Hence for every such disk $B$ it holds that (with $a_{B}=0$ )

$$
\frac{1}{|B|_{\lambda}} \int_{B}|F(z)| d \sigma(z) \leqq C(d) \cdot \sup _{z \in U} M_{d}(z) \text {. }
$$

Thus we have an $M$ such that for every closed disk $B$ in $U$ there is a constant $a_{B}$ satisfying the condition

$$
\int_{B}\left|F(z)-a_{B}\right| d \sigma(z) \leqq M \cdot|B|_{\lambda},
$$

which implies that $F(z)$ belongs to $\mathrm{BMO}(U, \lambda)$, hence $f$ belongs to $\mathrm{BMO}(R, \lambda)$.

Corollary 1. If $R$ is compact, then $\mathrm{BMO}(R)$ is coincident with $\operatorname{BMO}(R, \lambda)$.

COROLLARY 2. If $R$ admits Green's functions, then every Green's potential $P^{\mu}(p)$ with measure $\mu$ having a compact support on $R$ belongs to $\operatorname{BMO}(R, \lambda)$. 
Proof. Since the support of $\mu$ is compact, $P^{\mu}(p)$ is bounded outside a compact set containing the support. Hence the assertion follows from Theorem 2 and 4.

In case that $R=U$, we can generalize Corollary 2 as follows.

Proposition 2. Let $\mu$ be a measure on $U$ with a finite total mass, then the Green's potential $P^{\mu}(z)$ on $U$ belongs to $\operatorname{BMO}(U, \lambda)$.

Proof. Set $F_{0}(z)=\log (1 /|z|)$ and $a_{B}(0)=\int_{B} F_{0}(z) d \sigma(z) /|B|_{\lambda}$, for every closed disk $B$ in $U$, then because $F_{0}(z)$ belongs to $\operatorname{BMO}(U, \lambda)$ by Corollary 2, there is an $M$ such that

$$
\int_{B}\left|F_{0}(z)-a_{B}(0)\right| d \sigma(z) \leqq M \cdot|B|_{\lambda}
$$

for every closed disk $B$ in $U$.

Next set $T_{w}(z)=(z-w) /(1-\bar{w} z), F_{w}(z)=F_{0} \circ T_{w}(z)$ and $a_{B}(w)=\int_{B} F_{w}(z) d \sigma(z) /|B|_{\lambda}$ $\left(=a_{T_{w}(B)}(0)\right)$, then it is clear that $a_{B}(w)$ is continuous and bounded for every given $B$. So $a_{B}=\int_{U} a_{B}(w) d \mu(w)$ is finite and we have

$$
\begin{aligned}
\int_{B}\left|P^{\mu}(z)-a_{B}\right| d \sigma(z) & \leqq \int_{B}\left(\int_{U}\left|F_{w}(z)-a_{B}(w)\right| d \mu(w)\right) d \sigma(z) \\
& =\int_{U}\left(\int_{T_{w}(B)}\left|F_{0}(z)-a_{T_{w}(B)}(0)\right| d \sigma(z)\right) d \mu(w) \\
& \leqq M \cdot|B|_{\lambda} \cdot \mu(U),
\end{aligned}
$$

which implies that $P^{\mu}(z)$ belongs to $\operatorname{BMO}(U, \lambda)$.

\section{REFERENCES}

[1] A. Baernstein II, Analytic functions of bounded mean oscillation, Aspects of contemporary complex analysis, Academic Press (1980), 3-36.

[2] W. K. Hayman and Ch. Pommerenke, On analytic functions of bounded mean oscillation, Bull. London Math. Soc. 10 (1978), 219-224.

[3] T. A. Metzger, On BMOA for Riemann surfaces, Canadian J. Math. 33 (1981), $1255-1260$.

[4] H. M. Reimann and T. Rychener, Funktionen beschränkter mittlerer Oszillation, Lecture Notes in Math. 487, Springer (1975), 141 pp.

[5] M. Tsujl, Potential theory in modern function theory, Chelsea (1975), $590 \mathrm{pp}$.

Department of Matiematics

KYOTO UNIVERSITY 